\title{
Spatially resolved properties of the grand-design spiral galaxy UGC 9837: a case for high-redshift 2-D observations
}

\author{
K. Viironen ${ }^{1,2}$, S. F. Sánchez ${ }^{1}$, E. Marmol-Queraltó ${ }^{1,2}$, J. Iglesias-Páramo ${ }^{3,1}$, D. Mast ${ }^{1,3}$, R. A. Marino ${ }^{4,1}$, \\ D. Cristóbal-Hornillos ${ }^{2,3}$, A. Gil de $\mathrm{Paz}^{4}$, G. van de Ven ${ }^{5}$, J. Vilchez $^{3}$, and L. Wisotzki ${ }^{6}$ \\ 1 Centro Astronómico Hispano Alemán, C/ Jesús Durbán Remón 2-2, 04004 Almeria, Spain \\ e-mail: kerttu@cefca.es \\ 2 Centro de Estudios de Física del Cosmos de Aragón (CEFCA), C/ Pizarro 1, 3ª 41001 Teruel, Spain \\ 3 Instituto de Astrofísica de Andalucía (IAA-CSIC), Glorieta de la Astronomía s/n, 18008 Granada, Spain \\ ${ }^{4}$ Departamento de Astrofísica y CC. de la Atmósfera, Universidad Computense de Madrid, 28040 Madrid, Spain \\ 5 Max Planck Institute for Astronomy, Königstuhl 17, 69117 Heidelberg, Germany \\ ${ }^{6}$ Astrophysical Institute Potsdam, An der Sternwarte 16, 14482 Postdam, Germany
}

Received 17 December 2010 / Accepted 22 November 2011

\begin{abstract}
Context. We carry out a detailed 2-D study of the ionised gas in the local universe galaxy UGC 9837. In nearby galaxies, like the galaxy in question here, the spatial distribution of the physical properties can be studied in detail, providing benchmarks for galaxy formation models.

Aims. Our aim is to derive detailed and spatially resolved physical properties of the ionised gas of UGC 9837. In addition, we derive an integrated spectrum of the galaxy and study how varying spatial coverage affects the derived integrated properties. We also study how the same properties would be seen if the galaxy was placed at a higher redshift and observed as part of one of the high- $z$ surveys. Methods. UGC 9837 was observed using the PMAS PPAK integral-field unit. The spectra were reduced and calibrated and the stellar and ionised components separated. Using strong emission line ratios of the ionised gas, the source of ionisation, the dust extinction, the star formation rate, the electron density, and the oxygen abundance derived from a total integrated spectrum, central integrated spectrum, and individual fibre spectra are studied. Finally, the same properties are studied in a spectrum whose spatial resolution is degraded to simulate high- $z$ observations.

Results. The spatial distribution of the ionised gas properties is consistent with inside-out growing scenario of galaxies. We also find that lack of spatial coverage would bias the results derived from the integrated spectrum leading, e.g., to an underestimation of ionisation and overestimation of metallicity, if only the centre of the galaxy was covered by the spectrum. Our simulation of high- $z$ observations shows that part of the spatial information, such as dust and SFR distribution would be lost, while shallower gradients in metallicity and ionisation strength would be detected.
\end{abstract}

Key words. galaxies: individual: UGC 9837 - galaxies: abundances - galaxies: stellar content - techniques: spectroscopic stars: formation

\section{Introduction}

The local universe galaxies are fundamental anchor points when studying the evolution of galaxies with cosmological time. Their apparent scale lengths are small enough to derive relatively highresolution information on their physical properties with the current technology. This information, in turn, provides constraints that any theory of galaxy evolution need to meet. The Lambdacold dark matter paradigm $(\Lambda \mathrm{CDM})$ is currently the most widely accepted model describing the formation of structures in the Universe. In the case of the spiral galaxies, this model leads to the so-called inside-out formation scenario of galaxies (see, e.g. White \& Frenk 1991; Mo et al. 1998; Kepner 1999), i.e. the galaxy bulge forms first and is followed by the slower process of disk formation. This scenario leads to various predictions about the galaxy structure, such as negative age gradients, negative gradients of the gas-phase heavy element abundances, and a decreasing amount of dust with increasing galactocentric distance. These predictions can be tested observationally.

Integral-field spectroscopy provides a powerful tool for studying the distribution of physical properties in well-resolved galaxies (e.g., Rosales-Ortega et al. 2010; Sánchez et al. 2011b). Previous works have explored the use of different integral-field spectrophotometers for a detailed study of nearby galaxies. In particular, the SAURON project (de Zeeuw et al. 2002) and its extension Atlas3D (Cappellari et al. 2011) are focused on the analysis of early-type galaxies and bulges of spirals at $z<0.01$, to study their kinematics and stellar populations. Due to the distance of their objects $\left(D_{L}<42 \mathrm{Mpc}\right)$ and the field of view (FOV) covered by the SAURON instrument $\left(33^{\prime \prime} \times 41^{\prime \prime}\right)$, this study is mainly restricted to the central part of galaxies. The study of nearby spiral galaxies has been addressed by the VENGA survey using the VIRUS-P spectrograph (32 nearby spiral galaxies, Blanc et al. 2010), the DiskMass Survey combining PPAK and the SparsePak spectrograph (146 nearly face-on galaxies, Bershady et al. 2010), and the PINGS survey (17 nearby disky galaxies, Rosales-Ortega et al. 2010), using the PPAK IFU. In particular, the mosaicking designed for the PINGS survey allowed us to map the $\mathrm{H}_{\text {II }}$ regions along the whole extension of the galaxies and to explore the 2-D metallicity structure of disks. The galaxies of our sample (Mármol-Queraltó et al. 2011) were selected in size to fit in the FOV of the PPAK instrument, and 
therefore, to map their physical properties in their whole extension in just one pointing.

We present here a detailed study of ionised gas in one galaxy, UGC 9837. This Local Universe $(z \sim 0.009)$ galaxy is positioned nearly face-on towards us, making it a good candidate for 2-D galactic disk studies. The data were observed as part of the PMAS/PPAK, one of the world's widest FOV IFU, survey of 48 nearby galaxies presented by Mármol-Queraltó et al. (2011). This survey was part of the preparatory phase of the CALIFA survey (Sánchez et al. 2011a, 2012), a much larger IFS survey comprising observations of 600 nearby galaxies.

We used our data to derive for UGC 9837 2-D distributions of: 1) local nebular reddening estimates based on the Balmer decrement; 2) oxygen abundance distributions based on a suite of strong line diagnostics incorporating reddening-corrected $\mathrm{H} \alpha$, $\mathrm{H} \beta,\left[\mathrm{O}_{\mathrm{II}}\right],\left[\mathrm{O}_{\mathrm{III}}\right],[\mathrm{N} \mathrm{II}]$, and [S $\left.\mathrm{II}\right]$ line ratios; 3) measurements of the ionisation structure in $\mathrm{H}$ II regions and diffuse ionised gas using the well-known and latest forbidden-line diagnostics in the oxygen and nitrogen lines; 4) the distribution of the surface star formation rate (SFR) traced by the $\mathrm{H} \alpha$ line intensity. The derived spatial distribution of these properties allows us to test the insideout growing scenario of galaxies.

In addition to the 2-D maps, we also study the integrated values of the above-mentioned properties. The advantage of integral-field spectroscopy in this respect is that the observed spectra can be combined to produce an integrated spectrum of the object, using the IFU as a large aperture spectrograph. The real average spectroscopic properties of the galaxy can be derived from this high signal-to-noise, integrated spectrum . This way no assumptions need to be made, in contrast to previous studies (based on long-slit spectroscopy, for example) that attempt to describe the average spectroscopic properties of the galaxy based on individual spectra taken in different regions. This way the integrated properties of the galaxies in the Local Universe can be compared with the corresponding properties of the unresolved high-redshift objects. The most similar approach would be the spectrum derived by using drift-scanning technique (e.g. Moustakas \& Kennicutt 2006a). The advantage of using an IFU with respect to the drift-scan technique is that all the spectra are obtained simultaneously and that the IFU spectra allows a comparison between the integrated and the spatially resolved properties of the galaxy.

Finally, based on our Local Universe data, we study how the spatial properties of ionised gas could be seen in observations at higher redshift, such as those carried out by SINS (Förster Schreiber et al. 2006) or MASSIV (Epinat et al. 2009; Queyrel et al. 2009) surveys with VLT/SINFONI.

The article is structured in the following way: Sect. 2 presents the galaxy UGC 9837; Sect. 3 describes the observations and data reduction; in Sect. 4 the data analysis is presented; in Sect. 5 the results are discussed and in Sect. 6 the summary and conclusion are given. Throughout the paper we adopt a standard $\Lambda$ CDM cosmology with $H_{0}=70.4, \Omega_{\mathrm{m}}=0.268$, and $\Omega_{\lambda}=0.732$.

\section{UGC 9837}

UGC 9837 is a spiral galaxy of magnitude 14.6 in the $g$ band, located at redshift $z \sim 0.009$, and morphologically classified as SAB(s)c (de Vaucouleurs et al. 1991). In addition to the optical (SDSS), it has previously been observed at other wavelengths: UV (GALEX, Gil de Paz et al. 2007), NIR (2MASS, Skrutskie et al. 2006), and FIR (IRAS, Beichman et al. 1988), see Table 1.

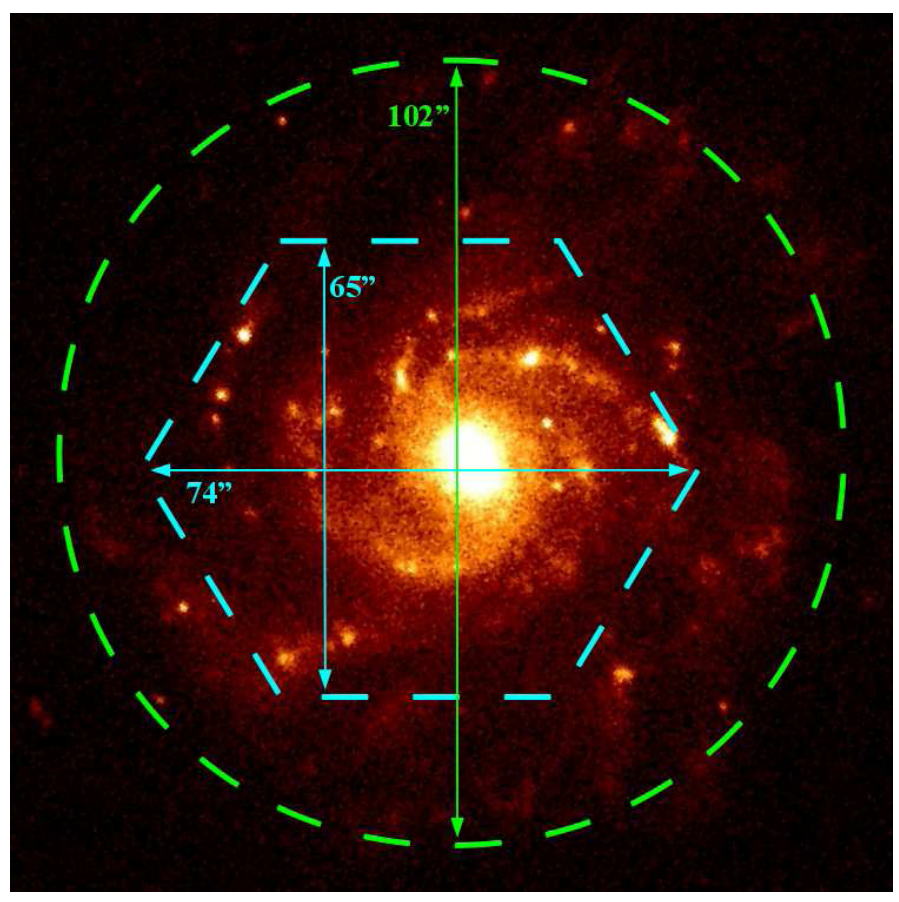

Fig. 1. SDSS ( $r$ band) image of UGC 9837. The cyan hexagon shows the approximate position of the PPAK pointing. The diameter of the green circle corresponds to the apparent diameter quoted in HyperLeda. North is up and east is left.

In addition, this galaxy was analysed as part of the DiskMass Survey (Bershady et al. 2010).

The distance to this galaxy has been estimated to be 41.2 Mpc (Tully 1988) with a distance modulus of 33.07 mag. The galaxy is extended $\left(2.0^{\prime} \times 1.8^{\prime}\right.$, Nilson 1973$)$ and nearly face-on, which permits the spatial properties of the disk to be fully studied. The total apparent corrected magnitude according to LEDA database is $13.65 \mathrm{mag}$, thus giving an absolute magnitude of $M_{B}=19.42 \mathrm{mag}$. Pohlen \& Trujillo (2006) report a bar of length $R \leq 9^{\prime \prime}(\sim 1.64 \mathrm{kpc}$ at the distance of the galaxy). These authors also find a break in the surface brightness profile at $R \sim 50^{\prime \prime}$, which almost corresponds to the optical diameter, $D_{25} \simeq 2^{\prime}$.

\section{Observations and data reduction}

UGC 9837 was observed on 23 June 2007. The night was not photometric and the extinction was high $\left(A_{\mathrm{V}}>0.2\right)$ and variable. During the night the seeing varied from 1 to $1.8^{\prime \prime}$. The observation were carried out using the $3.5 \mathrm{~m}$ telescope of the Calar Alto Observatory and the Potsdam Multi Aperture Spectrograph, PMAS (Roth et al. 2005) in the PPAK mode (Verheijen et al. 2004; Kelz et al. 2006).

Figure 1 shows the SDSS r-band image of UGC 9837 with the approximate position of the PPAK FOV superimposed. As can be seen, a single PPAK exposure covers approximately the whole area contained within the half the radius of the 25 mag $\operatorname{arcsec}^{-2}$ isophote. The centre of the PPAK FOV is slightly displaced from the optical centre of the galaxy in order to include some interesting $\mathrm{H}$ II regions to the east of the centre.

The PPAK fibre bundle consists of 331 science fibres of 2.7" diameter each, covering a total hexagonal FOV of $74 " \times 64^{\prime \prime}$ with a filling factor of $\sim 60 \%$. The sky is sampled by 36 additional fibres, distributed in six bundles of six fibres each, located following a circular distribution at about $90^{\prime \prime}$ from 
Table 1. Photometric properties of UGC 9837 as observed by different surveys at different passbands.

\begin{tabular}{cccccccc}
\hline \hline & SDSS & \multicolumn{2}{c}{ 2MASS } & \multicolumn{2}{c}{ IRAS } & \multicolumn{2}{c}{ GALEX } \\
\hline$u$ & $2.83 \mathrm{E}-03$ & $J$ & $1.03 \mathrm{E}-02$ & $12 \mu \mathrm{m}$ & $<5.82 \mathrm{E}-02$ & FUV & $2.73 \mathrm{E}-03$ \\
$g$ & $7.23 \mathrm{E}-03$ & $H$ & $1.20 \mathrm{E}-02$ & $25 \mu \mathrm{m}$ & $<6.05 \mathrm{E}-02$ & NUV & $3.29 \mathrm{E}-03$ \\
$r$ & $1.19 \mathrm{E}-02$ & $K$ & $9.37 \mathrm{E}-03$ & $60 \mu \mathrm{m}$ & $2.83 \mathrm{E}-01$ & & \\
$i$ & $1.45 \mathrm{E}-02$ & & & $100 \mu \mathrm{m}$ & $8.97 \mathrm{E}-01$ & & \\
$z$ & $1.51 \mathrm{E}-02$ & & & & & & \\
\hline
\end{tabular}

Notes. All the fluxes are given in Janskys.

the centre and at the edges of the central hexagon. The sky fibres are distributed among the science ones in the pseudo-slit in order to have a good characterisation of the sky. In addition there are 15 fibres used for calibration purposes.

The V300 grating was used, covering the wavelength range $\sim 3700-7100 \AA$, resulting in an effective spectral resolution of $\sim 8 \AA$ (FWHM). Three dithered $600 \mathrm{~s}$ exposures of UGC 9837 were taken following a pattern of $\triangle \mathrm{RA}= \pm 1.15^{\prime \prime}$ and $\triangle \mathrm{Dec}=$ $\pm 0.78^{\prime \prime}$ in order to have a complete coverage of the science FOV and to increase the spatial resolution. An additional $120 \mathrm{~s}$ exposures of the spectrophotometric standard $\mathrm{Hz} 4$ was obtained in order to perform the flux calibration. Also bias frames, arc lamp exposures, and sky exposures were taken for data reduction purposes.

Data reduction was carried out using R3D (Sánchez 2006), in combination with E3D (Sánchez 2004). The reduction consists of the standard steps for fibre-fed integral-field spectroscopy, including the bias subtraction, cosmic ray rejection, and extraction of the individual spectra by a Gaussian-suppression technique (Sánchez 2006) in order to minimise the effect of cross-talk between adjacent fibres. The extracted spectra were stored in a row-stacked-spectrum (RSS) file (Sánchez 2004) and were wavelength calibrated. Differences in the fibre-to-fibre transmission throughput were then corrected by comparing the wavelength-calibrated RSS science frames with the corresponding ones derived from the twilight sky exposures. Finally, the sky was subtracted and the spectra flux-calibrated.

After reducing the three individual pointings, the corresponding RSS frames were combined into a single frame using standard procedures included in R3D (see also Sánchez et al. 2011b; Mármol-Queraltó et al. 2011). First, each position table was combined in a single one by co-adding the differential offsets recorded by the telescope to each pointing. PMAS is equipped with a precise guiding system that allows us to perform relative offsets with an accuracy of $0.1^{\prime \prime}$. Then, a $10^{\prime \prime}$ radius aperture spectrum was extracted from the expected centre of the galaxy in each of the individual pointings, taking the offsets into account. These three spectra were compared, and if a difference larger than a 5\% was found, a rescaling was applied to match the intensity level of the first pointing. With this procedure, possible variations in the transparency between the different exposures is compensated for.

The resulting data were then spatially resampled in a regular grid data-cube, by interpolating the information in each individual spatial pixel at each individual wavelength. This is done by using the interpolation routines included in E3D (Sánchez 2004), by adopting a natural-neighbour non-linear interpolation scheme and a final pixel scale of $1^{\prime \prime}$ /pixel for the resulting data cube. The natural-neighbour routine adopted here is based on the Voronoi tessellation of a discrete set of spatial points. For each particular pixel within the new grid, the flux is assumed to be a weighted average of the n-adjacent ones:

$G(x, y)=\sum_{i=0}^{n} w_{i} F\left(x_{i}, y_{i}\right)$

where the $n$-neighbours are selected as the adjacent ones if the new pixel was included in the previous grid and a Voronoi tessellation was performed. The weights correspond to the fraction of area that the new tessellation will remove from the old one, if the new pixel is included. These weights are renormalised to conserve the integrated flux, by taking the number of adjacent pixels considered in each interpolation into account. It is important to note here that this interpolation does not require filling gaps between the fibres, which would be the case if only one single pointing procedure was adopted. As already shown in Sánchez et al. (2007), where this procedure was presented for the first time, the dithering guarantees a complete coverage of the FOV, and the interpolation routine resamples the data, without creating new values in areas not covered by the IFU.

Finally, the flux calibration of the resulting datacube was adjusted using the SDSS photometry. This was done because even though the relative flux calibration of our data should be reliable, there are many uncertainties that can affect the goodness of the absolute spectrophotometric calibration. The photon noise from the low surface brightness regions of the galaxy and the sky-background noise affect its quality. Also the sky subtraction entails inaccuracies. In addition, the night of the observations of UGC 9837 was not photometric. Recalibrating our data based on the SDSS photometry available for UGC 9837 is possible, because our IFS data allows us to carry our spectrophotometry over a wide enough aperture so that the comparison with a corresponding imaging photometry can be carried out without worrying about the PSF effects.

Of the five SDSS filters, our spectrum covers the pass bands of two, namely the $g^{\prime}$ and $r^{\prime}$ filters. We measured the counts in these SDSS images inside a 30" diameter aperture. Such an aperture is large enough to avoid problems in the compared photometry regarding the exact position of the aperture centre and the exact radius of the aperture. These counts were converted to flux following the counts-to-magnitude prescription in SDSS documentation $^{1}$. The corresponding spectrophotometry was then derived from our data, summing the flux of individual fibres inside a $30^{\prime \prime}$ diameter circle and convolving this spectrum with the SDSS $g^{\prime}$ and $r^{\prime}$ filter pass-bands. Using these two data pairs, an average scaling factor between the SDSS photometry and our spectrophotometry was calculated. This is shown in Fig. 2. The error in SDSS photometry is estimated to be $0.15 \mathrm{mag}$, including both the photon noise and the background-noise errors. The estimation is based on a statistical analysis in several different apertures of similar size on the background-subtracted $8^{\prime \prime} \times 8^{\prime \prime}$

\footnotetext{
${ }^{1}$ http://www.sdss.org/dr7/algorithms/fluxcal.html\# counts2mag
} 
Table 2. Measured and dereddened emission line fluxes (Flux, Flux_dr, respectively) of the SDSS spectrum, and central and total integrated our spectra (SDSS, cent, tot, respectively) of UGC 9837.

\begin{tabular}{lccccccc}
\hline \hline Line ID & $\lambda^{a}[\AA]$ & Flux (SDSS) & Flux_dr (SDSS) & Flux (cent) & Flux_dr (cent) & Flux (tot) & Flux_dr (tot) \\
\hline$[\mathrm{O}$ II $]$ & 3726.8 & - & - & $5.91(0.09)$ & $13.67(0.88)$ & $3.42(0.03)$ & $4.83(0.10)$ \\
$\mathrm{H} \gamma$ & 4340.5 & - & - & - & - & $0.43(0.02)$ & $0.51(0.02)$ \\
$\mathrm{H} \beta$ & 4862.0 & $1.00(0.06)$ & $1.00(0.06)$ & $1.00(0.06)$ & $1.00(0.06)$ & $1.00(0.02)$ & $1.00(0.02)$ \\
{$\left[\mathrm{O}_{\text {III }}\right]$} & 4959.5 & $0.23(0.02)$ & $0.22(0.02)$ & $0.17(0.02)$ & $0.15(0.01)$ & $0.45(0.01)$ & $0.44(0.01)$ \\
{$[\mathrm{O}$ III $]$} & 5007.8 & $0.68(0.05)$ & $0.65(0.05)$ & $0.50(0.04)$ & $0.45(0.04)$ & $1.37(0.02)$ & $1.31(0.02)$ \\
{$\left[\mathrm{N}_{\text {III }}\right]$} & 6548.1 & $0.55(0.02)$ & $0.42(0.03)$ & $0.65(0.02)$ & $0.30(0.02)$ & $0.24(0.01)$ & $0.17(0.01)$ \\
$\mathrm{H} \alpha$ & 6562.8 & $3.71(0.08)$ & $2.86(0.19)$ & $6.21(0.09)$ & $2.86(0.17)$ & $3.93(0.02)$ & $2.86(0.05)$ \\
{$\left[\mathrm{N}_{\text {II }}\right]$} & 6583.6 & $1.64(0.06)$ & $1.26(0.09)$ & $1.96(0.07)$ & $0.90(0.06)$ & $0.72(0.02)$ & $0.52(0.02)$ \\
{$\left[\mathrm{S}_{\text {II }}\right]$} & 6717.0 & $1.19(0.07)$ & $0.90(0.08)$ & $2.16(0.07)$ & $0.95(0.07)$ & $0.91(0.02)$ & $0.65(0.02)$ \\
{$\left[\mathrm{S}_{\mathrm{II}}\right]$} & 6731.0 & $0.91(0.05)$ & $0.69(0.06)$ & $1.70(0.07)$ & $0.74(0.06)$ & $0.67(0.02)$ & $0.48(0.02)$ \\
\hline
\end{tabular}

Notes. All fluxes are first corrected for the underlying stellar absorption and then normalised to $F(\mathrm{H} \beta)=1.0$ and their errors are given in parenthesis. ${ }^{(a)}$ Rest-frame.

Table 3. Physical properties of UGC 9837 derived from the SDSS spectrum, and central and total integrated our spectra (SDSS, cent, tot, respectively).

\begin{tabular}{lccc}
\hline \hline Property & SDSS & Cent & Tot \\
\hline $\log ([\mathrm{N}$ II $] / \mathrm{H} \alpha)$ & $-0.35 \pm 0.02$ & $-0.50 \pm 0.02$ & $-0.74 \pm 0.01$ \\
$\log \left(\left[\mathrm{O}_{\mathrm{III}}\right] / \mathrm{H} \beta\right)$ & $-0.39 \pm 0.03$ & $-0.70 \pm 0.04$ & $0.312 \pm 0.006$ \\
$\log (U)$ & - & $-4.21 \pm 0.04$ & $-3.473 \pm 0.009$ \\
$\mathrm{c}_{\beta}$ & $0.38 \pm 0.09$ & $1.13 \pm 0.08$ & $0.46 \pm 0.03$ \\
$A_{\mathrm{V}}^{\operatorname{gas}}[\mathrm{mag}]$ & $0.82 \pm 0.20$ & $2.43 \pm 0.18$ & $1.00 \pm 0.06$ \\
$12+\log (\mathrm{O} / \mathrm{H})(\mathrm{O} 3 \mathrm{~N} 2, \mathrm{Pe} 04)$ & $8.68 \pm 0.27$ & $8.68 \pm 0.27$ & $8.46 \pm 0.26$ \\
$12+\log (\mathrm{O} / \mathrm{H})(\mathrm{S} 2 \mathrm{~N} 2, \mathrm{Vi} 07)$ & $8.42 \pm 0.21$ & $8.25 \pm 0.19$ & $8.13 \pm 0.17$ \\
$12+\log (\mathrm{O} / \mathrm{H})(\mathrm{Pi} 10)$ & - & $8.36 \pm 0.16$ & $8.40 \pm 0.11$ \\
$S F R\left[M_{\odot} \mathrm{yr}^{-1}\right]$ & 0.06 & 0.13 & 2.48 \\
\hline
\end{tabular}

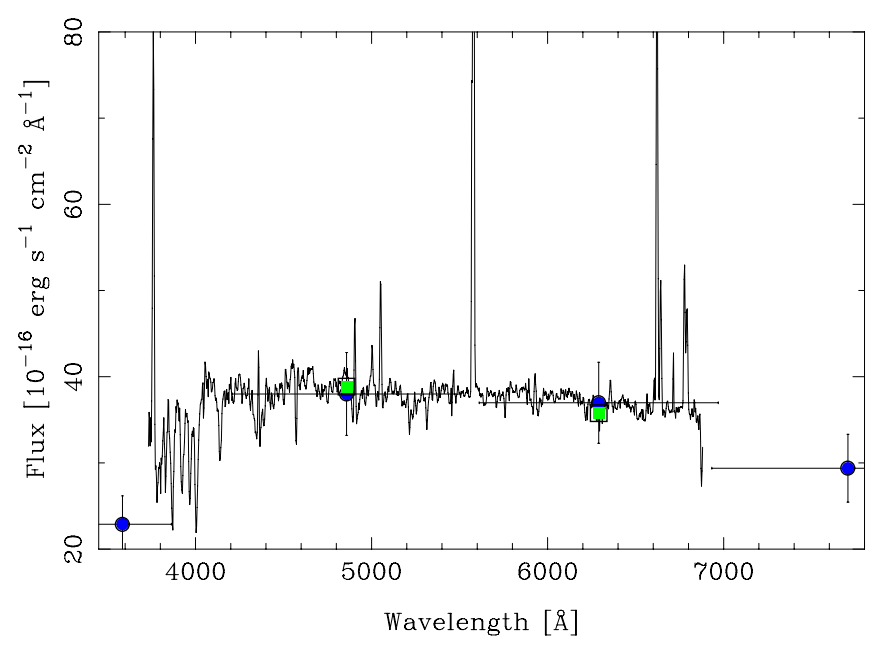

Fig. 2. The flux recalibration based on SDSS photometry. Our spectrum of the $30^{\prime \prime}$ diameter central region of UGC 9837, scaled by the average offset between it and the SDSS $g^{\prime}$ and $r^{\prime}$ band photometry, is shown as a black line. The SDSS $u^{\prime}, g^{\prime}, r^{\prime}$, and $i^{\prime}$, band photometry are shown as blue dots and our spectrophotometry before rescaling at $g^{\prime}$ and $r^{\prime}$ bands as green squares.

post-stamp images. Our spectrum was then recalibrated using the resulting average scaling factor (0.94), and the new calibrated spectrum was used in the following data analysis.

\section{Data analysis}

The first step in extracting any physical information from the data set is to identify the emission lines and to decouple their emission from the underlying stellar population. After this, the emission line spectrum is analysed using standard equations for ionised gas.

\subsection{Deriving the emission line fluxes}

Population synthesis was used to model and subtract the stellar continuum. This technique results in emission-line measurements corrected (to first order) for stellar absorption. Many different techniques are now available to perform such a decoupling. The one applied here is the one used in the analysis of the PINGS sample (Rosales-Ortega et al. 2010), and the details of this process are described in Sánchez et al. (2011b). Briefly, the scheme followed decoupling the stellar population, and the emission lines are the following: i) a set of emission lines is identified from the integrated spectrum; ii) for each spectrum in the data set, the underlying stellar population is fitted by a linear combination of a grid of single stellar populations (SSP), after correcting for the appropriate systemic velocity and velocity dispersion (including the instrumental dispersion which dominates the total observed dispersion when the grating V300 is used), and taking the effects of dust attenuation into account. The models were created using the MILES templates (Vazdekis et al. 2010) with three different ages (17.8, 1.0, and 0.09 Gyr) and two metallicities $(Z=0.004$ and 0.03$)$. Prior to the linear fitting, the nebular and sky emission lines are masked; iii) the fitted stellar population is subtracted from the original spectrum to get a residual pure emission-line spectrum; iv) finally, the intensities for each detected emission line are derived. We note that the adopted SSP template library has an impact on the derived properties of the SSP. It is actually not suitable for reliably 

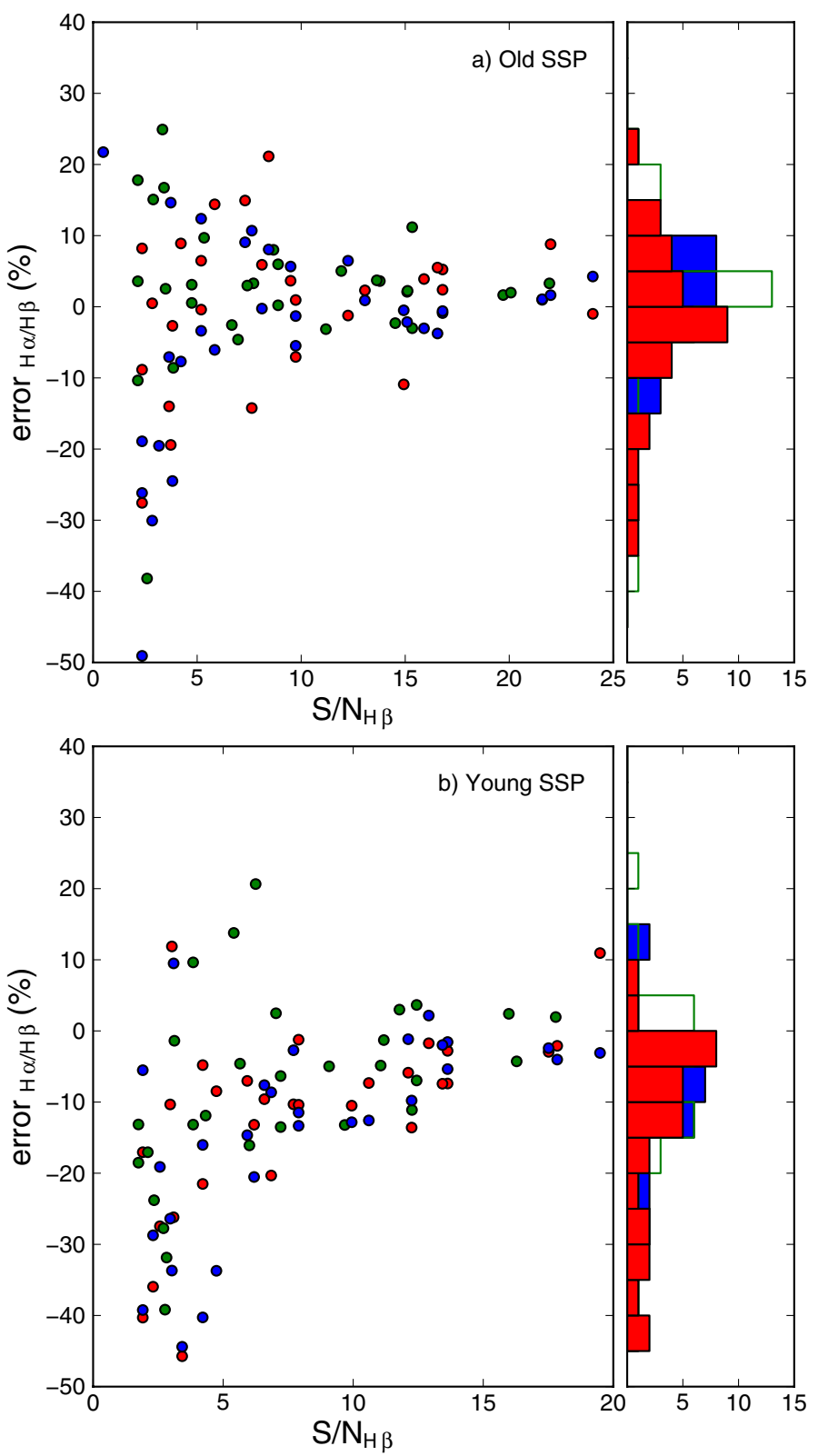

Fig. 3. Error in the derived $\mathrm{H} \alpha / \mathrm{H} \beta$ ratio as a function of the $\mathrm{S} / \mathrm{N}$ of the $\mathrm{H} \beta$ line for a young (1 Gyr, top) and old (17.78 Gyr, bottom) SSP with three different dust values, $A_{\mathrm{V}}=0.4$ (red), 0.5 (green), and 0.6 (blue).

deriving the SSP properties, but has little effect on the derived properties of the ionised gas (Mármol-Queraltó et al. 2011).

Next, the individual emission-line fluxes were measured in each spectrum by considering three spectral window regions: 1) $\left[\mathrm{O}_{\text {III }}\right] 3727 \AA$, 2) from $\mathrm{H} \gamma$ to $[\mathrm{O}$ III] $5007 \AA$, and 3) from $\left[\mathrm{N}_{\text {II }}\right] 6548 \AA$ to $\left[\mathrm{S}_{\text {II }}\right] 6731 \AA$. The windows were selected so that they cover all the nebular emission lines detected in the integrated spectrum. A simultaneous multi-component fitting was performed using a single Gaussian function (for each emission line contained within each window) plus a low-order polynomial (to describe the local continuum and to simplify the fitting procedure) using FIT3D (Sánchez et al. 2006). The central redshifted wavelengths of the emission lines were fixed, and since the FWHM is dominated by the instrumental resolution, the widths of all the lines were fixed as well. This procedure decreases the number of free parameters and increases the accuracy of the deblending process (when required). Line intensity

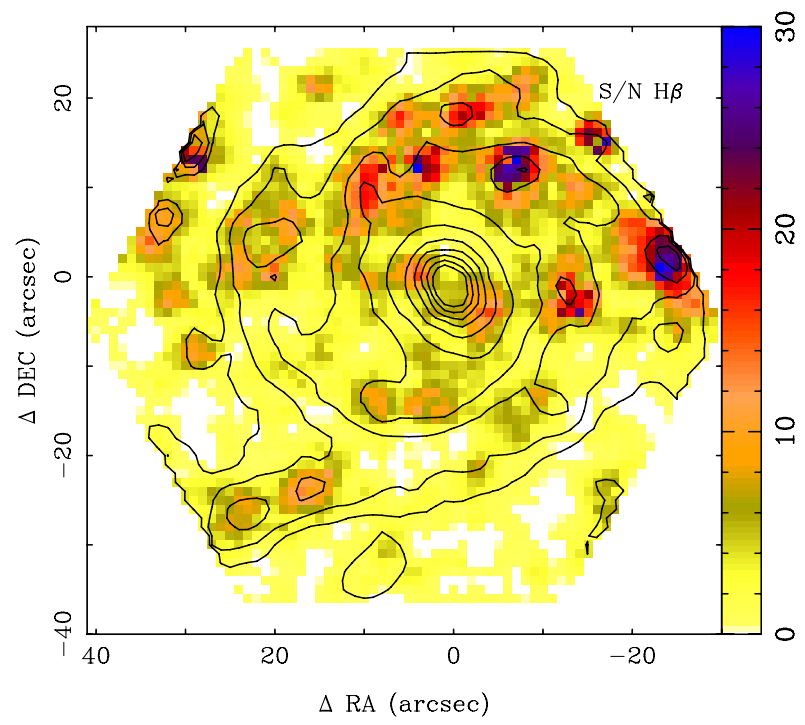

Fig. 4. 2-D map of the signal-to-noise ratio of the $\mathrm{H} \beta$ line. Contours correspond to the $V$-band emission derived from the datacube at the flux levels: $0.025,0.035,0.053,0.077,0.105,0.137,0.172,0.210,0.251$, and $0.295 \times 10^{16} \mathrm{erg} \mathrm{s}^{-1} \mathrm{~cm}^{-2}$.

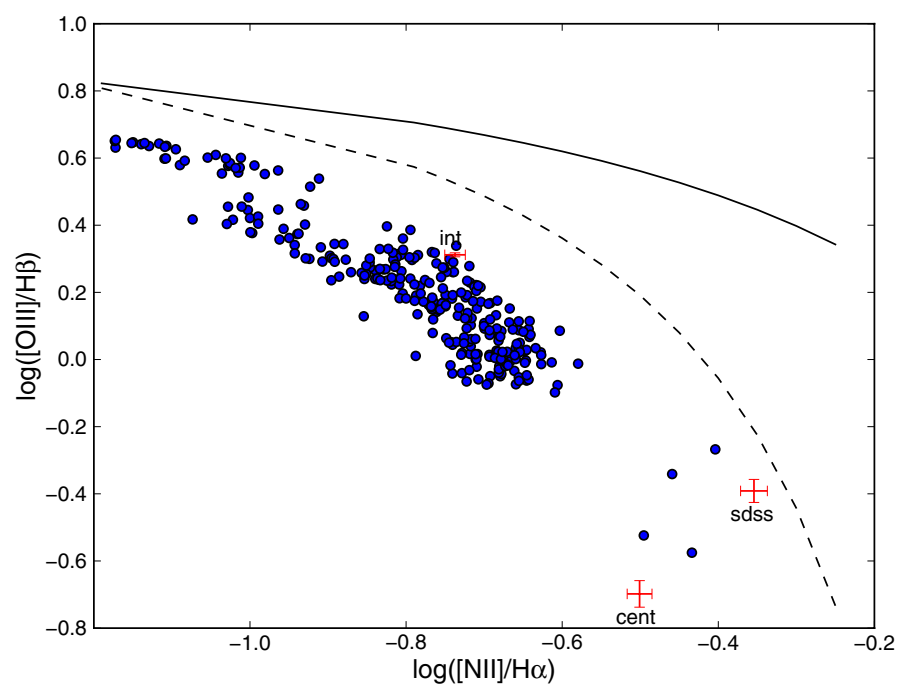

Fig. 5. The $\mathrm{N} 2 \mathrm{O} 3$ diagnostic diagram for our central spectrum (cent), the SDSS spectrum (sdss), the total integrated our spectrum (int), and the individual fibre-spectra of signal-to-noise ratio $>5 \sigma$ and $\mathrm{H} \alpha$ line intensity $>3 \times 10^{-16} \mathrm{erg} \mathrm{s}^{-1} \mathrm{~cm}^{-2} \operatorname{arcsec}^{-2}$ (blue dots). The solid and dashed lines separate the zones of starburst galaxies (below the lines) and AGNs (above the lines) as defined by Kewley et al. (2001) and Kauffmann et al. (2003), respectively.

fluxes were then measured by integrating the observed intensity of each line.

For the error analysis, an average continuum rms of the original spectrum was calculated and added by bootstrapping method to the $\mathrm{SSP}+$ gas model spectrum provided by the fitting program. This was repeated 100 times and each time the fitting was done on the resulting spectrum. The sigma of the median absolute deviation of these 100 simulations was then assigned as the error in the measured emission/absorption line fluxes.

The following experiment was carried out to test to what extent the decoupling procedure used affects the derived emission line fluxes. Different known emission line spectra (32 spectra derived from different parts of M74) were summed to a model 


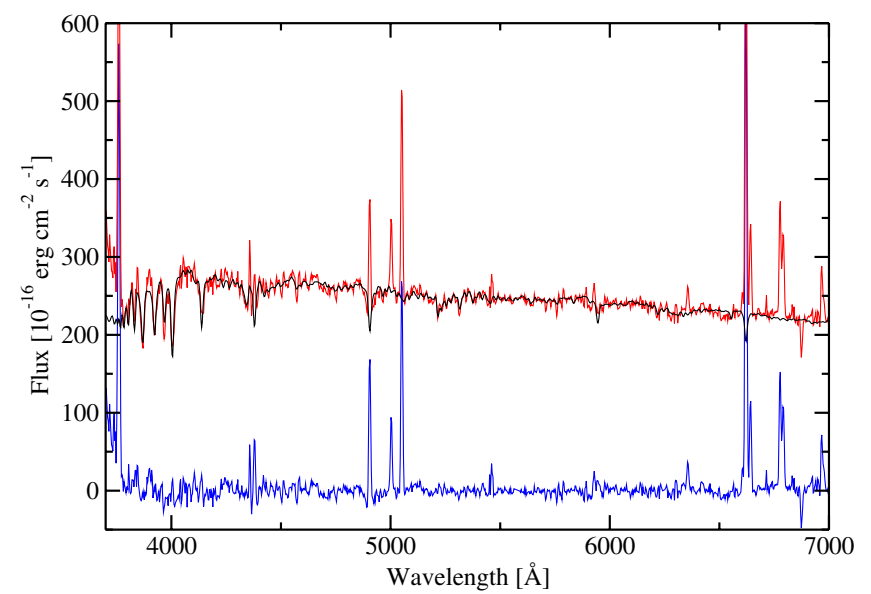

Fig. 6. The integrated spectrum of UGC 9837. The total integrated spectrum is shown in red, the best fitted combination of SSP models in black, and the spectrum of the gas only in blue.

spectra of both young (1 Gyr) and old (17.78 Gyr) SSP of Vazdekis et al. (2010), assuming three values of dust, $A_{\mathrm{V}}=0.4$, 0.5 , and 0.6 . The resulting spectra were then decoupled using the decoupling procedure introduced above, and the emission line fluxes measured. This was repeated 50 times by adding random noise to the spectrum to get an idea of the involved errors. The derived emission line fluxes were then compared with the corresponding original fluxes. Figure 3 shows the resulting difference (original - measured) in the measured $\mathrm{H} \alpha / \mathrm{H} \beta$ flux ratio. We see that the errors in the case of the young SSP are somewhat bigger than in the case of the old SSP, but in both cases the errors are reasonable, $\leq 20 \%$ for $\mathrm{S} / \mathrm{N}>5$. In the case of the young SSP one is also biased to underestimate the $\mathrm{H} \beta$ emission flux due to underlying strong absorption. This bias can be expected to affect the extinction map derived in Sect. 5.2 from $\mathrm{H} \alpha / \mathrm{H} \beta$ ratio. The $\mathrm{H} \alpha$ line map should not be strongly affected due to the strength of the $\mathrm{H} \alpha$ emission, while the rest of the maps are not affected by the bias because line ratios are used in their derivation. We show in Fig. 4 the 2-D map of the signal-to-noise ratio $(\mathrm{S} / \mathrm{N})$ of the $\mathrm{H} \beta$ line, so that the reader can have an idea of the expected spatial distribution of the errors associated to the decoupling procedure of ionised gas and stellar populations. In the centre of the galaxy and along the arms the $\mathrm{S} / \mathrm{N}$ is generally $>5$.

\subsection{Methods}

The emission line fluxes derived above reflect the physical properties of the galaxy. Its dust extinction can be derived from the $\mathrm{H} \alpha / \mathrm{H} \beta$ line ratio. Throughout the paper, we have adopted the extinction law of Cardelli et al. (1989) with $R_{\mathrm{V}}=3.1$. We assumed the Balmer decrement for case $\mathrm{B}$ recombination of ionised gas of temperature $10^{4} \mathrm{~K}$ and density of $100 \mathrm{~cm}^{-3}$ (Osterbrock 1989), i.e., $I(\mathrm{H} \alpha) / I(\mathrm{H} \beta) \sim 2.86$, as the unextincted expected value.

The nature of the ionisation is explored based on the $\log \left(\left[\mathrm{N}_{\mathrm{II}}\right] / \mathrm{H} \alpha\right)$ vs. $\log ([\mathrm{O} \mathrm{III}] / \mathrm{H} \beta)(\mathrm{N} 2 \mathrm{O} 3)$ diagnostic diagram of Baldwin et al. (1981) (see also Veilleux \& Osterbrock 1987). This diagnostic is insensitive to dust extinction, since the needed emission lines are very close in wavelength. This diagram allows the ionisation dominated by star-formation to be separated from the one dominated by an active galactic nucleus (AGN, see e.g., Kewley et al. 2001; Kauffmann et al. 2003).

The degree of the ionisation is traced by the ionisation parameter $U$. The lines available in our spectrum allow us to derive the ionisation parameter from the ratio $\left[\mathrm{O}_{\mathrm{II}}\right] /\left[\mathrm{O}_{\mathrm{III}}\right]=$ $\lambda 3727 /(\lambda 4959+\lambda 5007)$ using the formula (Díaz et al. 2000):

$\log U=-0.80 \log \left(\left[\mathrm{O}_{\text {II }}\right] /[\mathrm{O}\right.$ III $\left.]\right)-3.02$.

The electron density is derived from the [S II] $6717 \AA / 6731 \AA$ doublet ratio by assuming a typical electron temperature for starforming regions, $T_{\mathrm{e}}=10000 \mathrm{~K}$ (e.g. Osterbrock 1989).

For oxygen abundance determination, we use two empirical calibrations: O3N2 $=\log \left\{([\mathrm{O}\right.$ III $\left.] \lambda 5007 / \mathrm{H} \beta) /\left(\left[\mathrm{N}_{\mathrm{II}}\right] \lambda 6583 / \mathrm{H} \alpha\right)\right\}$ indicator of Pettini \& Pagel (2004) and the new calibration relations of Pilyugin et al. (2010) based on strong emission lines of oxygen, nitrogen, and sulphur. These calibration relations are given separately to three classes of $\mathrm{H}$ II regions: cool, warm, and hot, and the criteria how to classify the H II regions is given in Pilyugin et al. (2010). Even though we are not studying individual $\mathrm{H}_{\mathrm{II}}$ regions here, we used the same criteria to decide the equation for deriving the oxygen abundance from each spectrum. Finally, we compared the results from the empirical calibrations to the ones derived from the model based $\log \left(\mathrm{H} \alpha /\left[\mathrm{S}_{\mathrm{II}}\right] \lambda \lambda 6717+6731\right)$ vs. $\log \left(\mathrm{H} \alpha /\left[\mathrm{N}_{\mathrm{II}}\right] \lambda 6583\right), \mathrm{S} 2 \mathrm{~N} 2$, indicator of Viironen et al. (2007). Instead of more commonly used methods, such as the R23 index (Kewley \& Dopita 2002), we chose to use the O3N2 and S2N2 indicators because the SDSS spectrum with which we compare our data does not cover the [O II $] \lambda 3727$ line.

Finally, the star formation rate (SFR) of the galaxy is studied based on its $\mathrm{H} \alpha$ line intensity. The extinction corrected line fluxes are transformed to absolute luminosities and the corresponding SFRs are then derived by adopting the classical relation between the SFR and the luminosity of the $\mathrm{H} \alpha$ line (Kennicutt 1998).

\section{Results}

This section presents the results of the data analysis introduced above and carried out for integrated and spatially resolved spectra of UGC 9837.

\subsection{Integrated spectra}

To create an integrated spectrum, all the individual fibre spectra were convolved with the Johnson $V$ filter pass band, and only those spectra with fluxes greater than $10^{-18} \mathrm{erg} \mathrm{cm}^{-2} \mathrm{~s}^{-1}$ were summed. This was done in order to not to consider the data of the fibres that do not contain much of a signal or do not contain any signal at all as the fibres sample the regions where the intrinsic flux from the galaxy is low or zero (e.g. the borders of the galaxy, intra-arm regions). A similar approach was adopted in the analysis of the integrated spectrum of NGC 628, presented by Sánchez et al. (2011b), within the PINGS survey. We derive two integrated spectra, one covering only the central parts of the galaxy and another covering the whole data cube, and compare the physical properties derived from these spectra. For comparison, we carry out similar analysis for the SDSS spectrum, which covers only a small central region of the galaxy.

To derive the central spectrum, the entire pixels inside a $6^{\prime \prime}$ diameter are summed. The diameter of the SDSS spectrum aperture is $3^{\prime \prime}$. Since we are only interested in seeing quantitatively the differences in the physical properties derived in the centre as compared to those derived when (almost) the whole galaxy is covered, we did not try to exactly match the SDSS spectrum aperture because it would require interpolation (and the corresponding errors) because our pixel size is $1^{\prime \prime}$. In addition, we do not know the exact position of the SDSS aperture. 
The total integrated spectrum of UGC 9837 was created summing the spectra of all the fibres (fulfilling the $V$-band flux criterion above). This spectrum, with the ionised and stellar components separated, is shown in Fig. 6.

Once the integrated spectra are created, the stellar and ionised components are separated and emission lines measured and analysed as described in Sect. 4. Table 2 lists the emission lines and their fluxes with the corresponding errors in the SDSS spectrum, in our central, and in the total integrated spectrum. Table 3 lists the physical properties with their corresponding errors derived for UGC 9837 from the three above-mentioned spectra. The errors in the oxygen abundance include, in addition to the the total errors propagated from the errors in our data, the errors in the models themselves: 0.25 dex for the O3N2 method (Pettini \& Pagel 2004), 0.075 dex for the new calibrations of Pilyugin et al. (2010), and $0.15 \mathrm{dex}$ as indicated by Viironen et al. (2007) for the S2N2 diagnostics. In addition, the N2O3 diagram is shown in Fig. 5. The derived densities for the total integrated spectrum, central our spectrum, and the SDSS spectrum, respectively, are $N_{\mathrm{e}}=63 \pm 52,144 \pm 125$, and $107 \pm 173 \mathrm{~cm}^{-3}$. The errors are large because these low densities are at the lower limit where the $\left[\mathrm{S}_{\mathrm{II}}\right]$ ratio is hardly sensitive to the density anymore.

A clear difference is encountered when comparing the location of the integrated total spectrum with the central spectra in the N2O3 diagram, the former pointing to higher excitation than the latter. This is not surprising considering that the total integrated spectrum includes the high excitation star-forming regions of the spiral arms. Despite this difference, all the three spectra are located in the zone of star formation in this diagram. Also the ionisation parameter $U$ indicates higher ionisation for the total spectrum than the central one. Because the SDSS spectrum does not cover the $\left[\mathrm{O}_{\mathrm{II}}\right] \lambda 3727$ line, the degree of ionisation could not be derived from the SDSS spectrum. The extinction coefficient, $A_{\mathrm{V}}$, derived from the total integrated spectrum is lower than the corresponding central value. The value derived from our central spectrum is about three times the value derived from the SDSS spectrum. In principle this could happen if in the SSP/ionised gas decoupling of our lower resolution data, the $\mathrm{H} \beta$ absorption was underestimated. However, this would lead to a systematically higher values of all emission line fluxes (normalised to $\mathrm{H} \beta$ ) in our spectrum than for the SDSS spectrum. This is not the case, as seen in Table 2. Thus, we explain the difference in the two extinction values by short spatial scale variations of extinction, as the aperture sizes of the two spectra differ, and we do not know the exact position of the SDSS spectrum as respect to our spectrum. In Sect. 5.2 we show that there are significant spatial variations in extinction just near the centre of the galaxy.

There are clear shifts in the oxygen abundances derived using the different methods, the values derived from the S2N2 indicator being the smallest, and the ones derived from the O3N2 indicator the highest. These biases probably derive from the fact that in the integrated spectrum, the fluxes of different lines can come from physically different zones of the galaxy, with different ionising clusters and metallicities. Also, the S2N2 method, the one giving the lowest abundances, is not yet calibrated in a consistent manner with the other bright-line methods. Finally, the presence of diffuse ionised gas (DIG) can modify the final line ratios. Moustakas \& Kennicutt (2006b) show that a strong DIG would increase both the N2 and $\mathrm{S} 2$ ratios, but less so the higher ionisation $\mathrm{O} 3$ ratio. As a result, both $\mathrm{S} 2 \mathrm{~N} 2$ and $\mathrm{O} 3 \mathrm{~N} 2$ indicators would slightly overestimate the metallicity. However, as the integrated spectrum is dominated by bright $\mathrm{H}_{\text {II }}$ complexes, emitting strongly in $\mathrm{O} 3$, the effect of DIG in the abundance derived using O3N2 indicator is not necessarily significant (see, for example García-Benito et al. 2010). In the new calibration relations of Pilyugin et al. (2010), the metallicity is calculated as

$$
\begin{aligned}
12+\log (\mathrm{O} / \mathrm{H})=a_{0}+a_{1} P & +a_{2} \log \mathrm{O} 3 \\
& +a_{3} \log (\mathrm{N} 2 / \mathrm{O} 2)+a_{4} \log (\mathrm{S} 2 / \mathrm{O} 2)
\end{aligned}
$$

where

$$
P=\frac{\mathrm{O} 3}{\mathrm{O} 3+\mathrm{O} 2} \quad \text { and } \quad \mathrm{O} 2=[\mathrm{O} \Pi] \lambda \lambda 3727+3729 .
$$

The effect of DIG on the relation is not discussed, but considering that its emission causes S2 and N2 to increase, while O2, and especially O3, are less affected (Moustakas \& Kennicutt 2006b), probably the presence of DIG leads to a slight overestimation of metallicity when this relation is used.

When comparing the total and central spectra, the oxygen abundances of the former derived from the O3N2 (Pettini \& Pagel 2004) and S2N2 (Viironen et al. 2007) diagnostics lead to a lower oxygen abundance than for the corresponding values derived from the central spectra. This could be expected, since negative metallicity gradients are generally present in spiral galaxies. However, the abundances derived from the Pilyugin et al. (2010) calibration relations do not show such a trend, giving roughly the same result for the two spectra. In all cases the derived abundance is lower than the solar oxygen abundance $(12+\log (\mathrm{O} / \mathrm{H})=8.7$, Scott et al. 2009).

We derive an integrated $S F R$ of $\sim 2.4 M_{\odot} /$ year. It is close to the values derived for spiral galaxies and consistent with the picture of an unperturbed galaxy evolution (e.g., Usui et al. 1998). As expected, being an additive property, the star formation in the more reduced aperture of the central spectra is lower. When normalised by the aperture of each spectra, the SFR derived from the SDSS spectrum is actually the highest and then decreases when the aperture increases. This can be understood as in the larger aperture spectra the spectra of high star-forming spiral arms is mixed with the spectra of intra-arm regions with low-level diffuse $\mathrm{H} \alpha$ emission.

\subsection{Two-dimensional distribution of the gas properties}

The real power of 2-D spectroscopy resides in its capacity to map the physical properties of objects along their spatial extensions. We create the emission line maps for UGC 9837 from the final reduced datacube by carrying out the fitting procedure described in Sect. 4.1 for each individual spectrum. For each map, the pixels with a flux $<0.2 \times 10^{-16} \mathrm{erg} \mathrm{cm}^{-2} \mathrm{~s}^{-1}$ at the corresponding wavelength are masked, in order to retain only the high $\mathrm{S} / \mathrm{N}$ data $(\gtrsim 5 \sigma)$. When creating maps of emission line ratios, only those locations are considered where all the involved lines are above this selected minimum flux. Practically speaking, a map of any detected emission line, or a combination of them, could be derived from the individual fibre spectra. Here we present a number of maps corresponding to the most important emission lines and derived quantities of interest.

As the dominant gas ionisation mechanism giving rise to the emission lines in this galaxy is due to hot (OB) stars, the $\mathrm{H} \alpha$ line intensity map, shown in the top-left panel of Fig. 7, traces the star-forming regions of the galaxy. The map shows a clear spiral structure with $\mathrm{H}$ II complexes of different sizes and morphologies along the spiral arms. The brightest sources are located in the northern arms. Also a clear presence of diffuse emission is visible along the spiral arms and in the intra-arms region. 

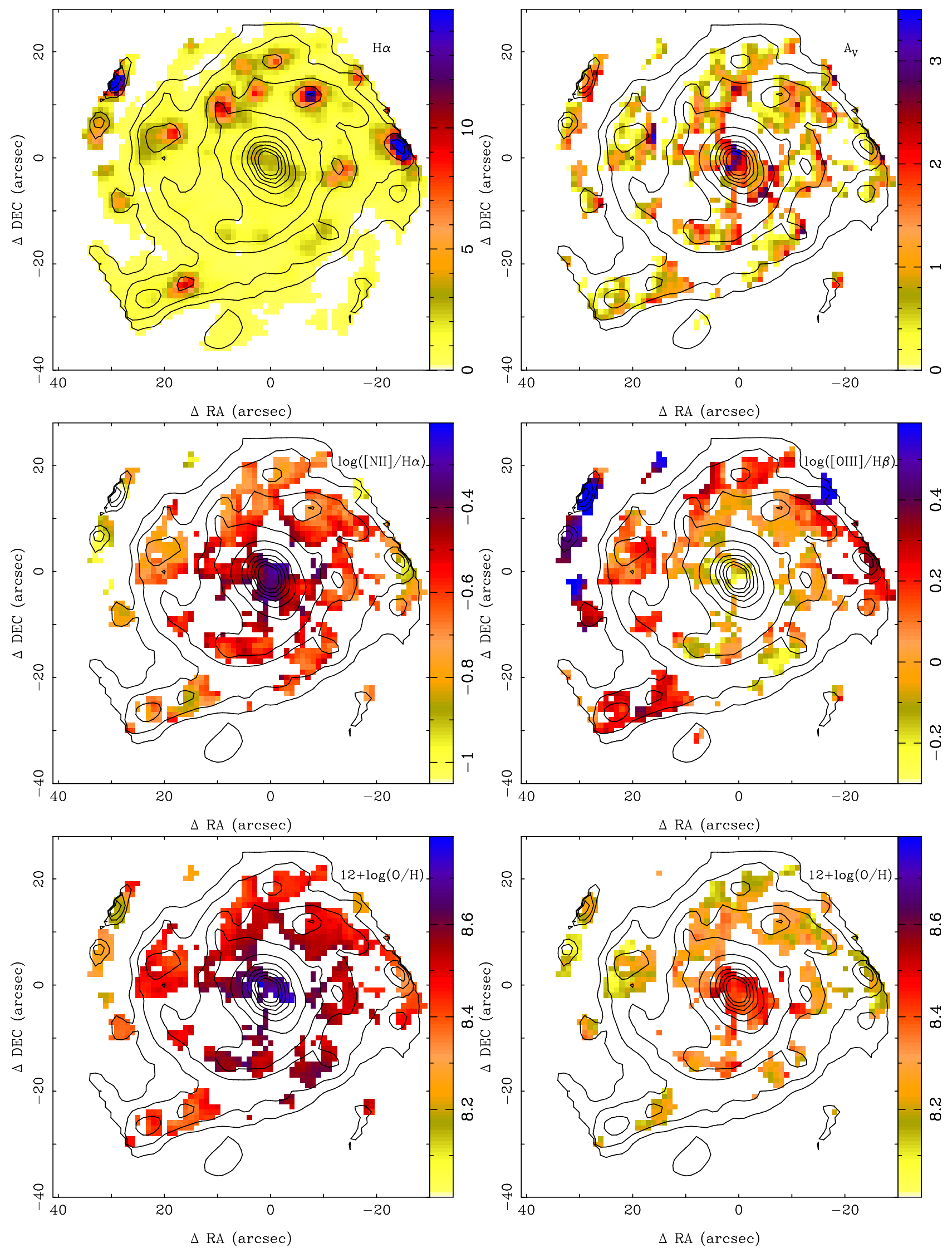

Fig. 7. Top left: $\mathrm{H} \alpha$ line intensity map in units of $10^{-16} \mathrm{erg} \mathrm{s}^{-1} \mathrm{~cm}^{-2} \operatorname{arcsec}^{-2}$. Top right: dust extinction map of the ionised gas. Middle left: $\log \left(\left[\mathrm{N}_{\mathrm{II}}\right] / \mathrm{H} \alpha\right)$ and middle right: $\log \left(\left[\mathrm{O}_{\mathrm{III}}\right] / \mathrm{H} \beta\right)$ diagnostic line-ratio maps. Bottom left: distribution of the oxygen abundance according to the O3N2 indicator of Pettini \& Pagel (2004) and bottom right: S2N2 relation of Viironen et al. (2007). The contours represent the $V$-band image derived from the data-cube and show the flux levels $0.025,0.035,0.053,0.077,0.105,0.137,0.172,0.210,0.251$, and $0.295 \times 10^{-16} \mathrm{erg} \mathrm{s}^{-1} \mathrm{~cm}^{-2}$. 


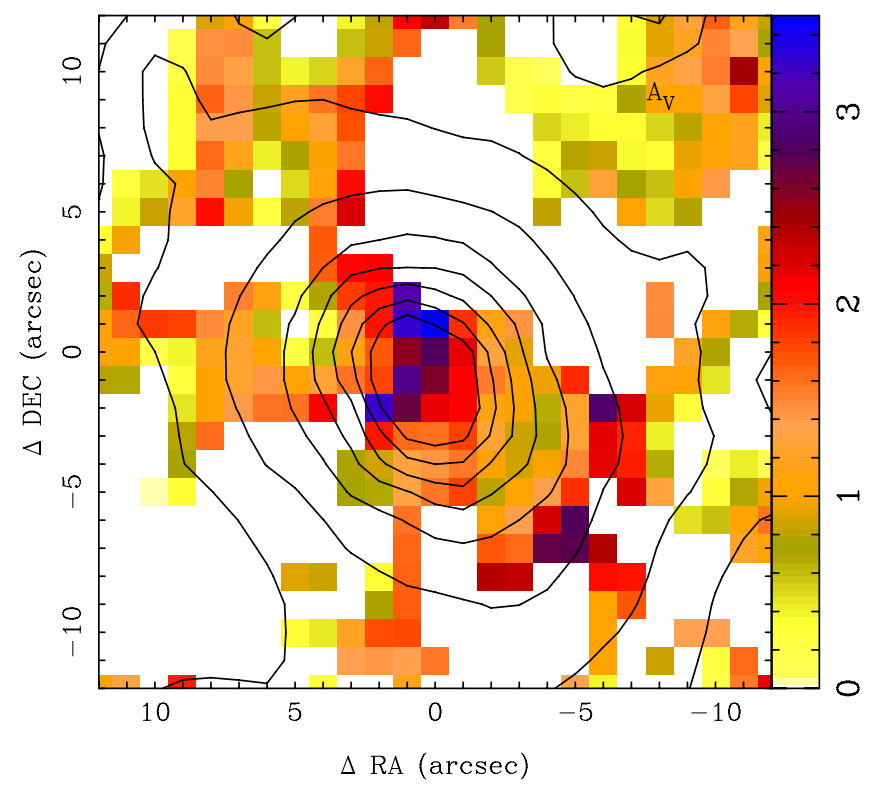

Fig. 8. Closer look at the extinction variation (see Fig. 7 top right) in the centre of the galaxy.

The dust extinction was derived from $\mathrm{H} \alpha / \mathrm{H} \beta$ line ratio, as in the previous section, and is shown in top right of Fig. 7 in terms of $A_{\mathrm{V}}$. The dust extinction is very variable, but as a general trend it is strongest in the bulge and then follows the spiral arms. In the areas populated by young stars, i.e. expected in the spiral arms, the extinction may be overestimated by $\leq 20 \%$ (see Figs. 3 and 4.) A strong short-scale variation in extinction in the centre of the galaxy is visible (see also Fig. 8), as already indicated in Sect. 5.1 by the differences between the extinction values derived from our and SDSS central spectra.

The dust extinction map described above is used to deredden the rest of the line maps, and only the pixels covered by the dust map, in addition to the data on the lines in question, are considered. The middle panels of Fig. 7 shows the diagnostic line-ratio maps $\log ([\mathrm{N}$ II $] / H \alpha)$, and $\log ([\mathrm{O}$ III $] / H \beta)$ (Veilleux $\&$ Osterbrock 1987). The radial gradient is clear, the ionisation hardening towards the edge. This is indicated by the strengthening of the $[\mathrm{O} I I I] / \mathrm{H} \beta$ ratio towards the edge, while the $[\mathrm{N} \mathrm{II}] / \mathrm{H} \alpha$ ratio decreases. No clear trend along the spiral arms is visible. As shown in Fig. 5 the values of both ratios at any $\mathrm{H} \alpha$ emitting location in the galaxy are consistent with ionisation produced by hot OB stars according to both Kewley et al. (2001) and Kauffmann et al. (2003).

Next we studied the spatial distribution of the oxygen abundance as for the integrated spectra above using for brevity only the O3N2 indicator of Pettini \& Pagel (2004) and S2N2 diagnostics of Viironen et al. (2007). The resulting maps are shown in the bottom panels of Fig. 7. As the comparison of the central and integrated spectra above already hints, in both maps the oxygen abundance is clearly strongest in the centre and decreases towards the edge, showing a gradient of $\sim-0.4$ dex, but O3N2 about 0.3 dex offset from $\mathrm{S} 2 \mathrm{~N} 2$.

\subsection{Simulations of observations at higher redshifts}

In this section we study how the above maps would look if the galaxy would be located at a higher redshift $(z \simeq 1-2)$ and observed as part of surveys such as SINS (Förster Schreiber et al. 2006) or ongoing MASSIV (Epinat et al. 2009; Queyrel et al. 2009), both using VLT/SINFONI. For this we have used a code adapted from the one used in Eikenberry et al. (2006). The simulated spatial scale is $0.125^{\prime \prime} /$ pix, and the data was depixeled accordingly, summing only entire pixels. Considering the original and simulated pixel sizes there is no difference in the depixelisation for simulated redshifts of 1.5 (typical for MASSIV) or 2.2 (typical for SINS). Next the flux was rescaled as

$F=F_{0}\left(\frac{d_{\text {orig }}}{d_{\text {simul }}}\right)^{2}\left(\frac{1+z_{\text {orig }}}{1+z_{\text {simul }}}\right)$,

where $d_{\text {orig }}$ and $d_{\text {simul }}$ are the original and simulated luminosity distances, respectively, and $z_{\text {orig }}$ and $z_{\text {simul }}$ the original and simulated redshifts. Finally, we assumed seeing conditions of $0.6^{\prime \prime}$ and convolved the data with 2-D Gaussian kernel of $0.6^{\prime \prime}$ FWHM. We accepted for the resulting datacube only those pixels where the convolution does not rely on the zeropadding used outside the image area. We have not added any noise to the simulated data because it depends on the observation strategy (e.g. integration time). However, in real observations at high redshift one would generally expect lower $\mathrm{S} / \mathrm{N}$ than in the lower- $z$ observations. We neither considered the instrumental response of the simulated instrument at the wavelength where the line fluxes would be shifted at the simulated redshift nor considered whether the galaxy is intrinsically bright enough to be detected by the instrument at this redshift. Thus, to summarise, our simulation shows what kind of data could be obtained on a galaxy like UGC 9837 if it was located at high redshift and detected by an IFU of $0.125^{\prime \prime}$ pixel scale in typical observing conditions of $0.6^{\prime \prime}$ seeing. At the level of this simulation, the picture would change only slightly if instrument like Keck/OSIRIS with $0.1^{\prime \prime}$ pixel scale was simulated, while if a smaller pixel scale (which generally leads to a smaller FOV) was assumed, the result would be improved.

In Fig. 9 we show the simulated maps (at $z=2.2$ ) derived from the maps in Fig. 7. Since there would be no differences in the depixelisation and we have not touched the $\mathrm{S} / \mathrm{N}$ or considered the instrumental response, the corresponding maps at $z=1.5$ would be equal but have higher flux. Comparing the maps in Figs. 7 and 9 we see that, in the high-redshift simulation, the spatial information about the SFR (H $\alpha$ line map) is lost except for a gradient of stronger $\mathrm{H} \alpha$ emission towards the north, where the brightest $\mathrm{H}$ II regions are located. The information about the distribution of dust is lost completely.

The combined effect of the last two not only prevents us from deriving information about the SFR distribution along the galactic disk, but also leads to a bias in the level of SFR itself. As discussed in Sect. 5.2, the spatial distribution of the extinction by dust is very variable. In general, it is strongest in the centre and then follows the spiral arms. The loss of this information leads to underestimating absolute line fluxes, and SFR, in the dust-rich parts of the galaxy, while the opposite is true for the less dusty parts.

For the relative line fluxes, the bluer line intensities are underestimated compared to the redder lines in the dusty regions, especially in the centre and in the spiral arms. For this reason, we warn against using dust-dependent indicators, such as emission line ratios of lines with a large wavelength difference or absolute line fluxes, if the distribution of dust extinction is not known.

The gradients in $\log \left(\left[\mathrm{N}_{\mathrm{II}}\right] / \mathrm{H} \alpha\right)$ and $\log \left(\left[\mathrm{O}_{\mathrm{III}}\right] / \mathrm{H} \beta\right)$ line ratio maps, as well as in the $12+\log (\mathrm{O} / \mathrm{H})$, can still be roughly appreciated in the simulated maps. However, the gradients appear shallower in the simulated maps than for the original ones. Shallower gradients can be expected because the spatial resolution of the simulated maps is worse so that zones of higher and 
A\&A 538, A144 (2012)
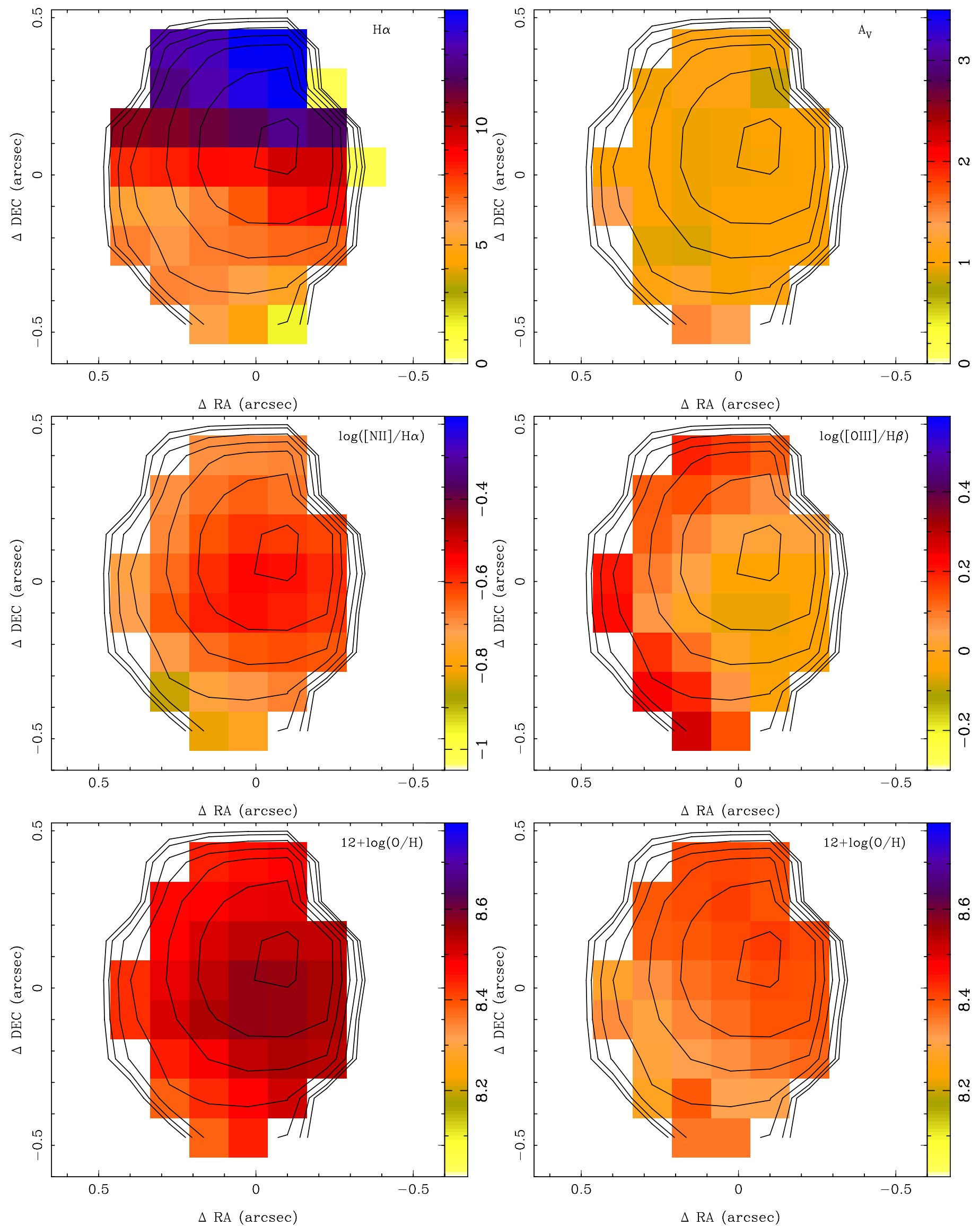

Fig. 9. The same maps (but in units of $0.5 \times 10^{-24} \mathrm{erg} \mathrm{s}^{-1} \mathrm{~cm}^{-2} \operatorname{arcsec}^{-2}$ ) as in Fig. 7 but as they would be seen by VLT/SINFONI if the UGC 9837 was placed at redshift $z=2.2$. 
lower ionisation and metallicity are mixed. This has an effect in the interpretation of the abundance gradients derived from observations with limited spatial resolution.

The inside-out growing scenario of galactic disks predicts the presence of negative metallicity gradients in spiral galaxies. However, different chemical evolution models disagree on the time variation of these gradients. Some models predict that the gradients steepen with time (e.g. Chiappini et al. 2001) while other models predict the opposite (e.g. Fu et al. 2009). Recently, Queyrel et al. (2012) have published a study of metallicity gradients in a sample of galaxies at $z \sim 1.2$ based on SINFONI data. They find both negative, zero, and positive gradients, explaining the last by interactions. If a gradient was detected, it was in all cases shallower than the typical gradients of the nearby galaxies. Based on our simulation above, we argue that the gradients they measure are instead lower limits of the real abundance gradients of these galaxies.

\section{Summary and conclusions}

We have carried out a detailed study of the ionised gas of UGC 9837 using the 2-D data obtained with PMAS/PPAK (Mármol-Queraltó et al. 2011). We studied the integrated and spatially resolved properties of the ionised gas, deriving the nature of ionisation, ionisation parameter, oxygen abundance, SFR, and dust extinction $\left(A_{\mathrm{V}}\right)$ of the galaxy. First we compared the ionised gas properties derived from a spectrum covering only the centre of the galaxy (derived from our data and a SDSS spectrum) with the corresponding properties derived from a spectrum covering (almost) the whole galaxy. Clear differences were encountered because the spectra sample different zones of the galaxy. We conclude that deriving global properties of the galaxy from a spectrum of only partial spatial coverage would lead to significant biases. We derived for the galaxy a total SFR of $\sim 2.4 M_{\odot} /$ year, which is consistent with the picture of an unperturbed galaxy evolution for this object (e.g., Usui et al. 1998). The integrated oxygen abundance derived using different strong line methods varied, but all diagnostics lead to a subsolar abundance.

Next, the spatially resolved properties of the ionised gas were studied. We found that the ionisation becomes harder with increasing distance from the centre of the galaxy, while negative gradients of both oxygen abundance and dust extinction were uncovered. In general, our results are consistent with an insideout growing scenario for disk galaxies, where the more evolved stellar populations and richer gas components with more dust are located in the centre of the galaxies, and the less evolved stellar populations and metal poor gas, with less dust grains and stronger SFR and harder ionisation, are located in the outer regions.

We also carried out a simulation of how the galaxy would be seen if it was placed at a higher redshift and observed as part of surveys such as MASSIV or SINS. In our simulation the spatial information about the SFR and dust distribution is lost, while radial gradients of ionisation strength and oxygen abundance can still be roughly distinguished. The gradients derived from the simulated data are shallower than the original (more realistic) gradients. These biases should be kept in mind when interpreting observations with limited spatial resolution. According to our Local Universe data, the dust distribution along the galactic disk can be very variable. If this information is lacking, dustindependent indicators should be used for the study of the galaxy in question. The metallicity gradients derived from data with poor spatial resolution, in turn, are lower limits of the actual ones.

Finally, we want to point out the importance of a statistical sample of 2-D data on nearby galaxies. The already existing data of the Mármol-Queraltó et al. (2011) sample, and ultimately the data on a large sample of Local Universe galaxies being obtained by ongoing CALIFA (Sánchez et al. 2011a, 2012) survey will allow analysis like the one presented here to be carried out in a statistical manner. This in turn will provide strong constraints on the theories of galaxy evolution and fix observational properties of galaxies in the Local Universe, which may have a potential impact on the interpretation of observed properties at higher redshifts.

Acknowledgements. We thank the Viabilidad, Diseño, Acceso y Mejora funding programme, ICTS-2009-10, of the Spanish Ministerio de Ciencia e Innovación, for the support given to this project. This paper makes use of the Sloan Digital Sky Survey data. Funding for the SDSS and SDSS-II has been provided by the Alfred P. Sloan Foundation, the Participating Institutions, the National Science Foundation, the U.S. Department of Energy, the National Aeronautics and Space Administration, the Japanese Monbukagakusho, the Max Planck Society, and the Higher Education Funding Council for England. The SDSS Web Site is http://www.sdss.org/. The SDSS is managed by the Astrophysical Research Consortium for the Participating Institutions. The Participating Institutions are the American Museum of Natural History, Astrophysical Institute Potsdam, University of Basel, University of Cambridge, Case Western Reserve University, University of Chicago, Drexel University, Fermilab, the Institute for Advanced Study, the Japan Participation Group, Johns Hopkins University, the Joint Institute for Nuclear Astrophysics, the Kavli Institute for Particle Astrophysics and Cosmology, the Korean Scientist Group, the Chinese Academy of Sciences (LAMOST), Los Alamos National Laboratory, the Max-PlanckInstitute for Astronomy (MPIA), the Max-Planck-Institute for Astrophysics (MPA), New Mexico State University, Ohio State University, University of Pittsburgh, University of Portsmouth, Princeton University, the United States Naval Observatory, and the University of Washington. Finally, we want to thank the anonymous referee for useful comments that helped us to improve the article considerably.

\section{References}

Baldwin, J. A., Phillips, M. M., \& Terlevich, R. 1981, PASP, 93, 5

Beichman, C. A., Neugebauer, G., Habing, H. J., Clegg, P. E., \& Chester, T. J. 1988, Infrared astronomical satellite (IRAS) catalogs and atlases, Vol. 1: Explanatory supplement

Bershady, M. A., Verheijen, M. A. W., Swaters, R. A., et al. 2010, ApJ, 716, 198 Blanc, G. A., Gebhardt, K., Heiderman, A., et al. 2010, in New Horizons in Astronomy: Frank N. Bash Symposium 2009, ed. L. M. Stanford, J. D. Green, L. Hao, \& Y. Mao, ASP Conf. Ser., 432, 180

Cappellari, M., Emsellem, E., Krajnović, D., et al. 2011, MNRAS, 413, 813

Cardelli, J. A., Clayton, G. C., \& Mathis, J. S. 1989, ApJ, 345, 245

Chiappini, C., Matteucci, F., \& Romano, D. 2001, ApJ, 554, 1044

de Vaucouleurs, G., de Vaucouleurs, A., Corwin, Jr., H. G., et al. 1991, Third Reference Catalogue of Bright Galaxies, ed. N. G. Roman, G. de Vaucouleurs, A. de Vaucouleurs, H. G. Corwin, Jr., R. J. Buta, G. Paturel, \& P. Fouqué

de Zeeuw, P. T., Bureau, M., Emsellem, E., et al. 2002, MNRAS, 329, 513

Díaz, A. I., Castellanos, M., Terlevich, E., \& Luisa García-Vargas, M. 2000, MNRAS, 318, 462

Eikenberry, S., Andersen, D., Guzman, R., et al. 2006, in SPIE Conf. Ser., 6269 Epinat, B., Contini, T., Le Fèvre, O., et al. 2009, A\&A, 504, 789

Förster Schreiber, N. M., Genzel, R., Lehnert, M. D., et al. 2006, ApJ, 645, 1062 Fu, J., Hou, J. L., Yin, J., \& Chang, R. X. 2009, ApJ, 696, 668

García-Benito, R., Díaz, A., Hägele, G. F., et al. 2010, MNRAS, 408, 2234

Gil de Paz, A., Boissier, S., Madore, B. F., et al. 2007, ApJS, 173, 185

Kauffmann, G., Heckman, T. M., Tremonti, C., et al. 2003, MNRAS, 346, 1055

Kelz, A., Verheijen, M. A. W., Roth, M. M., et al. 2006, PASP, 118, 129

Kennicutt, Jr., R. C. 1998, ARA\&A, 36, 189

Kepner, J. V. 1999, ApJ, 520, 59

Kewley, L. J., \& Dopita, M. A. 2002, ApJS, 142, 35

Kewley, L. J., Dopita, M. A., Sutherland, R. S., Heisler, C. A., \& Trevena, J. 2001, ApJ, 556, 121 
Mármol-Queraltó, E., Sánchez, S. F., Marino, R. A., et al. 2011, A\&A, 534, A8 Mo, H. J., Mao, S., \& White, S. D. M. 1998, MNRAS, 295, 319

Moustakas, J., \& Kennicutt, Jr., R. C. 2006a, ApJS, 164, 81

Moustakas, J., \& Kennicutt, Jr., R. C. 2006b, ApJ, 651, 155

Nilson, P. 1973, Nova Acta Regiae Soc. Sci. Upsaliensis Ser. V, 0

Osterbrock, D. E. 1989, Astrophysics of gaseous nebulae and active galactic nuclei (Univ. Science Books)

Pettini, M., \& Pagel, B. E. J. 2004, MNRAS, 348, L59

Pilyugin, L. S., Vílchez, J. M., \& Thuan, T. X. 2010, ApJ, 720, 1738

Pohlen, M., \& Trujillo, I. 2006, A\&A, 454, 759

Queyrel, J., Contini, T., Pérez-Montero, E., et al. 2009, A\&A, 506, 681

Queyrel, J., Contini, T., Kissler-Patig, M., et al. 2012, A\&A, in press, DOI: $10.1051 / 0004-6361 / 201117718$

Rosales-Ortega, F. F., Kennicutt, R. C., Sánchez, S. F., et al. 2010, MNRAS, 405, 735

Roth, M. M., Kelz, A., Fechner, T., et al. 2005, PASP, 117, 620

Sánchez, S. F. 2004, Astron. Nachr., 325, 167

Sánchez, S. F. 2006, Astron. Nachr., 327, 850

Sánchez, S. F., García-Lorenzo, B., Jahnke, K., et al. 2006, Astron. Nachr., 327,167
Sánchez, S. F., Cardiel, N., Verheijen, M. A. W., et al. 2007, A\&A, 465, 207 Sánchez, S. F., Kennicutt, R. C., Gil de Paz, A., et al. 2011a, Highlights of Spanish Astrophysics VI, Proc. of the IX Scient. Meet. Span. Astron. Society (SEA), ed. M. R. Zapatero Osorio, et al., 339

Sánchez, S. F., Rosales-Ortega, F. F., Kennicutt, R. C., et al. 2011b, MNRAS, 410,313

Sanchez, S. F., Kennicutt, R. C., Gil de Paz, A., et al. 2012, A\&A, 538, A8

Scott, P., Asplund, M., Grevesse, N., \& Sauval, A. J. 2009, ApJ, 691, L119

Skrutskie, M. F., Cutri, R. M., Stiening, R., et al. 2006, AJ, 131, 1163

Tully, R. B. 1988, Nearby galaxies catalog, ed. R. B. Tully

Usui, T., Saito, M., \& Tomita, A. 1998, AJ, 116, 2166

Vazdekis, A., Sánchez-Blázquez, P., Falcón-Barroso, J., et al. 2010, MNRAS, 404, 1639

Veilleux, S., \& Osterbrock, D. E. 1987, ApJS, 63, 295

Verheijen, M. A. W., Bershady, M. A., Andersen, D. R., et al. 2004, Astron. Nachr., 325, 151

Viironen, K., Delgado-Inglada, G., Mampaso, A., Magrini, L., \& Corradi, R. L. M. 2007, MNRAS, 381, 1719

White, S. D. M., \& Frenk, C. S. 1991, ApJ, 379, 52 